\title{
DESIGN AND SIMULATION OF HORN ANTENNA FOR GPR APPLICATION
}

\author{
Shilpa \\ Dept. of Electronics and Communication \\ RNSIT \\ Bengaluru, India \\ sshilpapatil12@gmail.com
}

\begin{abstract}
This paper presents horn antenna design in the X-band (8-12GHz) for GPR application. Horn is designed and simulated using CST-MW (Computer Simulation Technology - Microwave) studio that makes use of FDTD technique providing 3d EM field simulations. We describe horn design with higher directivity, lower VSWR and lower return loss in the desired frequency band. The designed antennas are fabricated, compared and verified with the simulated results.
\end{abstract}

Keywords-taper (flare); waveguide; aperture; E-plane; Hplane, directivity, Ground Penetrating RADAR (GPR), CST-MW studio.

\section{INTRODUCTION}

The detection and identification of subsurface objects such as landmines and unexploded ordinances (UXO) has become a serious issue in recent decades as they are causing problems of humanitarian and hence an interesting field of research. Antennas are the most important and crucial part of a GPR system to detect the subsurface objects. Antenna is defined as "a metallic device that radiates or receives electromagnetic waves or radio waves". RADAR works by transmitting and receiving electromagnetic (EM) waves from an antenna. Ground penetrating radar (GPR) is also termed as georadar, that applies electromagnetic waves to image the targets buried in the ground.

Many antennas have been designed in the past for GPR which include Vivaldi [1], bowtie [2] and dipole antennas. This paper presents the design of horn for GPR. Figure 1 is the GPR system with transmitting and receiving antenna.

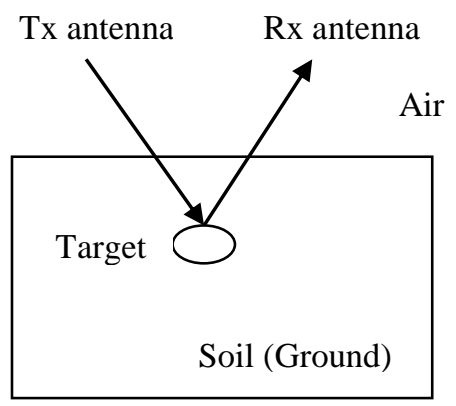

Fig. 1 GPR system

In GPR system, the transmitting antenna sends the electromagnetic waves continuously as it is moved close to the ground and the reflected waves are received by the receiving antenna. In the presence of the target, GPR senses electrical non uniformities of the target in the presence of a less conducting surrounding ground [3]. The changes in the soil

\author{
WG Cdr S.N.Sridhara \\ Dept. of Electronics and Communication \\ RNSIT \\ Bengaluru, India \\ sridhara.s.n@rnsit.ac.in
}

properties significantly affect the obtained reflections from the target.

Horn is treated as an aperture antenna. It is no more than a hollow pipe of different cross sections, which has been tapered to a larger opening. The type, direction and amount of taper (flare) have profound effect on the overall performance of the element as a radiator. The utilization of horn antennas is common in different fields such as RADAR, reflector feeds, detection systems, satellite tracking and communication dishes.

The paper is organized as follows. Section II provides the horn antenna design considerations. Section III provides the information on the software used. Simulation of the antenna is discussed in section IV and section $\mathrm{V}$ is the conclusion of the paper.

\section{DESIGN APPROACH}

Horn antennas are typically fed by a section of a waveguide. A simple construction, ease of excitation, versatility, large gain with wide bandwidth has made horn more advantageous compared to other antennas. Figure 2 is a pyramidal horn which is flared in both the directions. The radiation pattern is the combined effect of E-plane and $\mathrm{H}$-plane radiation patterns. Figure 3 and 4 gives the E-plane and $\mathrm{H}$ plane view of the horn antenna.

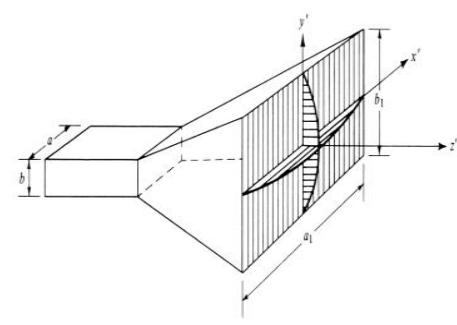

Fig. 2 Pyramidal horn

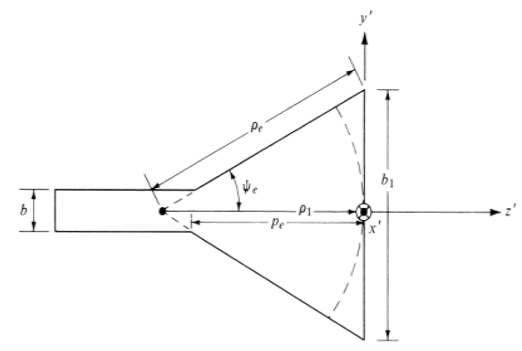

Fig. 3 E-plane view 


\section{Asia Pacific Journals}

\section{USING THE TEMPLATE}

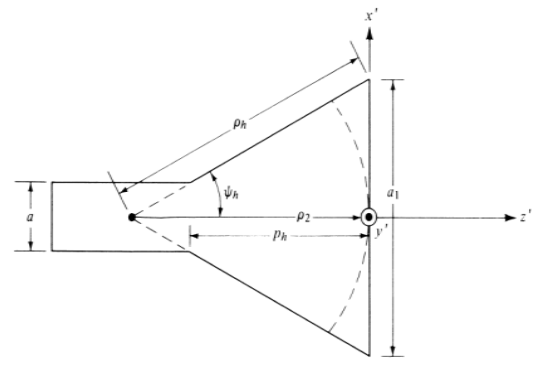

Fig. 4 H-plane view

\section{A. Horn design}

Horn antenna can be designed with few considerations such as knowing the desired gain $\mathrm{G}_{0}$ and the operating frequency. The design-equation for horn is as follows

$$
\left(\sqrt{2 \chi}-\frac{b}{\lambda}\right)^{2}(2 \chi-1)=\left(\frac{G_{0}}{2 \pi} \sqrt{\frac{3}{2 \pi}} \frac{1}{\sqrt{\chi}}-\frac{a}{\lambda}\right)^{2}\left(\frac{G_{0}{ }^{2}}{6 \pi^{3}} \frac{1}{\chi}-1\right)
$$

Step1: Find the value of $\chi$ for a desired gain $G_{0}$ using the equation

$$
\chi=\frac{G_{0}}{2 \pi \sqrt{2 \pi}}
$$

Step2: Once $\chi$ value is found, values of $\rho_{e}$ and $\rho_{h}$ are determined as follows

$$
\begin{gathered}
\frac{\rho_{e}}{\lambda}=\chi \\
\frac{\rho_{h}}{\lambda}=\frac{\mathrm{G}_{0}{ }^{2}}{8 \pi^{3}}\left(\frac{1}{\chi}\right)
\end{gathered}
$$

Step3: The corresponding values of a1 and b1 are determined as below

$$
\begin{gathered}
a 1=\frac{G_{0}}{2 \pi} \sqrt{\frac{3}{2 \pi \chi}} \lambda \\
b 1=\sqrt{2 \chi \lambda}
\end{gathered}
$$

Step4: Finally, the last step is to find the values of $P_{e}$ and $P_{h}$. To realize a pyramidal horn physically $P_{e}$ and $P_{h}$ must be equal.

$$
p_{e}=(b 1-b)\left[\left(\frac{\rho_{e}}{b 1}\right)^{2}-\frac{1}{4}\right]^{\frac{1}{2}}
$$

$$
p_{h}=(a 1-a)\left[\left(\frac{\rho_{h}}{a 1}\right)^{2}-\frac{1}{4}\right]^{\frac{1}{2}}
$$

\section{SOFTWARE CST-MWS}

CST-MWS (Computer Simulation TechnologyMicrowave Studio) is the industry standard for simulating 3-D full wave electromagnetic fields. Antenna design and complex antenna problems require computational electromagnetic (CEM) field for computation, where electromagnetic solvers are used that solve the subset of Maxwell's equation directly. CST consists of solvers both in frequency and time domain.

CST uses finite difference time domain (FDTD) method to solve electromagnetic problems. FDTD method represents the derivatives in Maxwell's curl equations by finite differences. FDTD has been proven to be very efficient for realizing GPR problems. FDTD technique is a second order accurate direct solution method for Maxwell's time dependent curl equations [4].

$$
\begin{aligned}
& \nabla \times H=J_{e}+\frac{\partial D}{\partial t} \\
& \nabla \times E=-J_{m}-\frac{\partial B}{\partial t}
\end{aligned}
$$

\section{A. FDTD algorithm flow}

To implement FDTD algorithm, the computational domain is divided into cubic cells as shown in figure 5 . The $\mathrm{E}$ and $\mathrm{H}$-field components at each cell are calculated from the finite difference expressions based on Yee's algorithm.

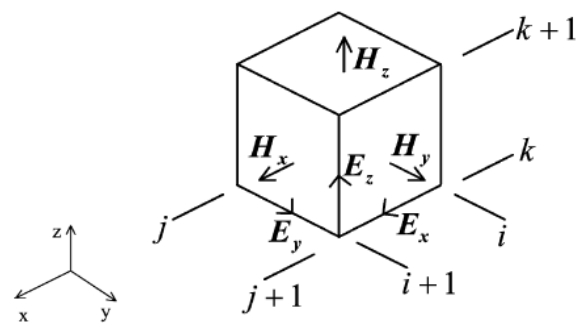

Fig. 5 FDTD Cell

Step1: Set problem space and define parameters.

Step2: Compute field coefficients.

Step3: Update magnetic field components at time instant $(n+0.5) \Delta t$.

Step4: Update electric field components at time instant $(n+1) \Delta t$

Step5: Apply boundary conditions.

Step6: Increment time step, $n \leftarrow n+1$. 


\section{Asia Pacific Journals}

Step7: Output data. If last iteration, stop else repeat from step3.

\section{SIMULATION RESULTS}

GPR RADARs can be of different types such as monostatic, quasi-static, bi-static and multi-static. The simulations are carried out considering monostatic GPR. Figure 6 shows the simulation setup for GPR using CST with horn antenna. The antenna is placed at a certain distance in air from the target in the soil. The antenna is sourced or triggered and hence the electromagnetic waves are transmitted from the antenna into the soil.

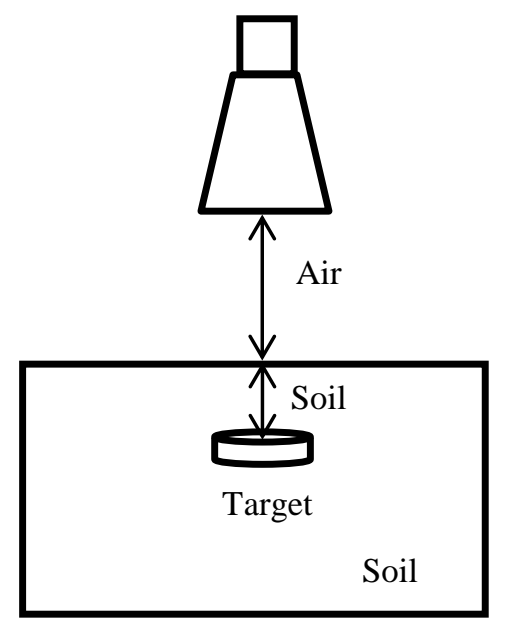

Fig. 6 Simulation setup

Figure 7 is the return loss of the horn antenna. When the transmitted waves hit any object or target in the soil there is a change in the property of the transmitted wave and hence the wave gets reflected which is known as return loss. Return loss is less than $-10 \mathrm{~dB}$ for the simulated antenna. Figure 7, 8 and 9 are the radiation patterns in two and three dimensional plots yielding a directivity of $20.59 \mathrm{dBi}$, angular width $(3 \mathrm{~dB})$ of 12.1 degree and side lobe level of $-13.2 \mathrm{~dB}$.

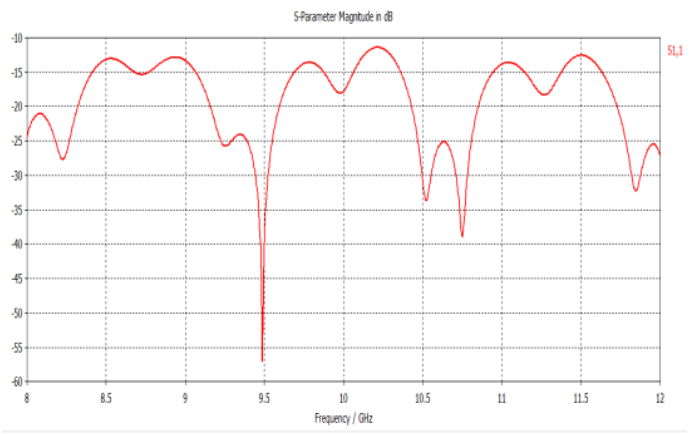

Fig. 6 Return loss

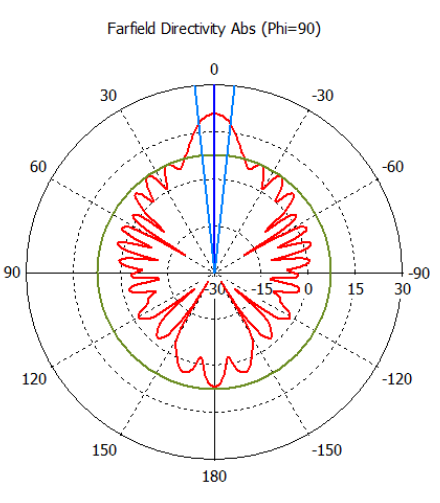

Theta / Degree vs. dBi

Fig. 7 Radiation pattern

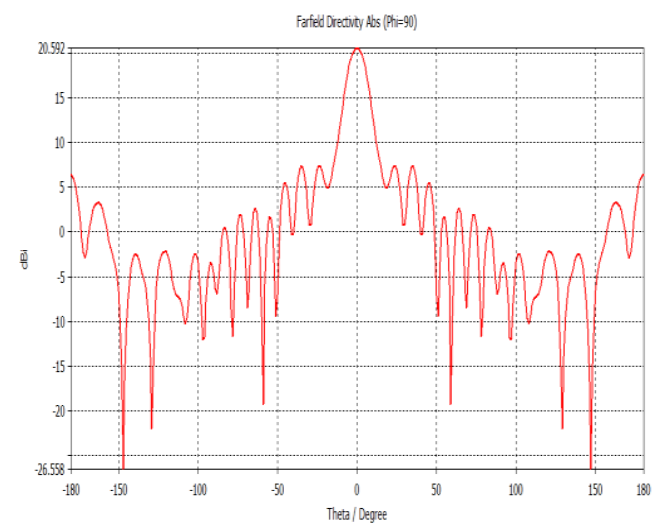

Fig. 8 Cartesian plot

Figure 7 is the radiation pattern around a complete $360^{\circ}$ rotation.

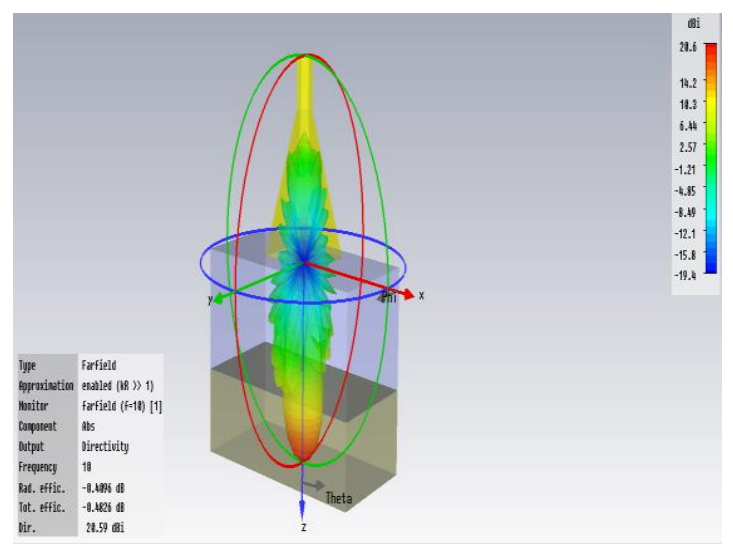

Fig. 9 3D plot of radiation pattern 


\section{Asia Pacific Journals}

TABLE I. SIMULATION RESULTS FOR VARYING SOIL(DRY) DEPTHS

\begin{tabular}{|c|c|c|c|c|}
\hline \multirow{4}{*}{ Air $=10 \mathrm{~cm}$} & $\begin{array}{c}\text { Soil } \\
\text { Depth(dry) }\end{array}$ & Directivity & $\begin{array}{c}\text { Penetration } \\
\text { depth }\end{array}$ & $\begin{array}{c}\text { Angular } \\
\text { width }\end{array}$ \\
\cline { 2 - 5 } & $10 \mathrm{~cm}$ & $20.28 \mathrm{dBi}$ & $8 \mathrm{~cm}$ & $13.6^{\circ}$ \\
\cline { 2 - 5 } & $30 \mathrm{~cm}$ & $19.56 \mathrm{dBi}$ & $24 \mathrm{~cm}$ & $15.3^{\circ}$ \\
\cline { 2 - 5 } & $50 \mathrm{~cm}$ & $19.27 \mathrm{dBi}$ & $38 \mathrm{~cm}$ & $15.5^{\circ}$ \\
& & & & \\
\hline
\end{tabular}

Table I gives the simulation results of the horn for the different soil depths. As the depth of the soil increases or if the target is placed farther from the soil surface, signal attenuation increases as it has to penetrate deeper. Deeper the penetration greater the attenuation of the signal and hence the directivity decreases as the target depth is increased. The simulation results predict that the presented horn can detect a target at a depth of about $38 \mathrm{~cm}$ from the soil surface. As the target depth increases directivity decreases and hence the beam width increases.

TABLE II. SIMULATION RESULTS FOR VARYING AIR DEPTHS

\begin{tabular}{|c|c|c|c|}
\hline \multirow{3}{*}{ Soil=20 cm } & Air depth & Directivity & $\begin{array}{c}\text { Penetration } \\
\text { depth }\end{array}$ \\
\cline { 2 - 4 } & $10 \mathrm{~cm}$ & $19.54 \mathrm{dBi}$ & $17 \mathrm{~cm}$ \\
\cline { 2 - 4 } & $20 \mathrm{~cm}$ & $19.33 \mathrm{dBi}$ & $16 \mathrm{~cm}$ \\
\cline { 2 - 4 } & $30 \mathrm{~cm}$ & $19.28 \mathrm{dBi}$ & $12 \mathrm{~cm}$ \\
\hline
\end{tabular}

Table II provides the simulation results for different distances of antenna from the soil. Closer the antenna to the ground, higher the signal penetration leading to higher directivity. Thus there is a higher possibility of object detection. As the antenna is closer to the ground the target can be easily detected. As the distance of the antenna from the ground increases the time taken to detect the object goes on increasing.

Horn antenna is commonly used antenna for subsurface probing. One of the important requirements of GPR system is ultra wide bandwidth. Broadband properties of the designed horn can be increased further by loading the inside of the horn with a dielectric material. Figure 10 is the return loss for dielectric loaded antenna. Figure 11, 12 and 13 are two dimensional and two dimensional plots of dielectric loaded horn for GPR with a directivity of $20.3 \mathrm{dBi}$, angular width $(3$ $\mathrm{dB}$ ) of 14.1 degree and side lobe level of $-11.1 \mathrm{~dB}$.

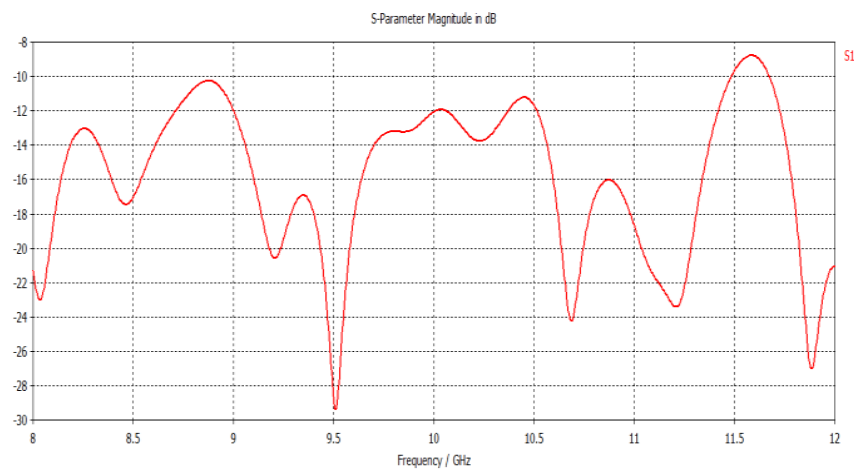

Fig. 10 Return loss

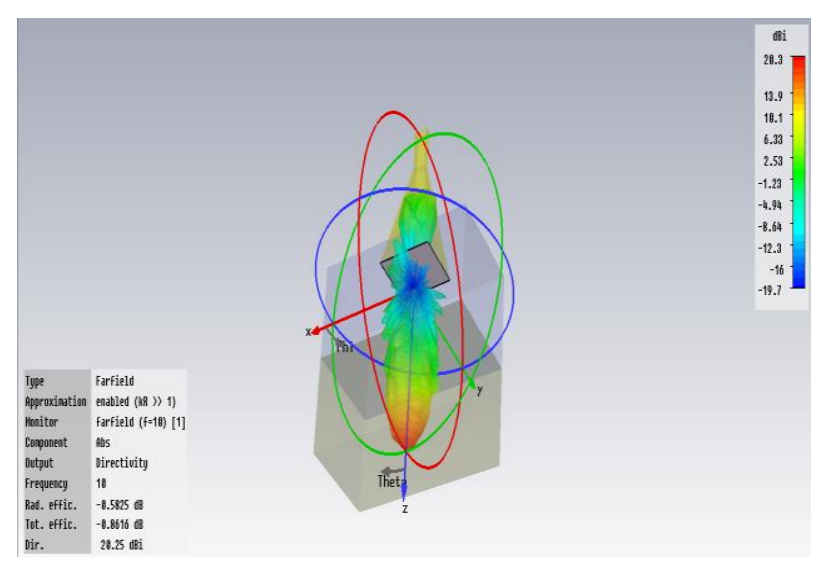

Fig. $113 \mathrm{D}$ plot of radiation pattern with dielectric loading at $\mathrm{f}=10 \mathrm{GHz}$

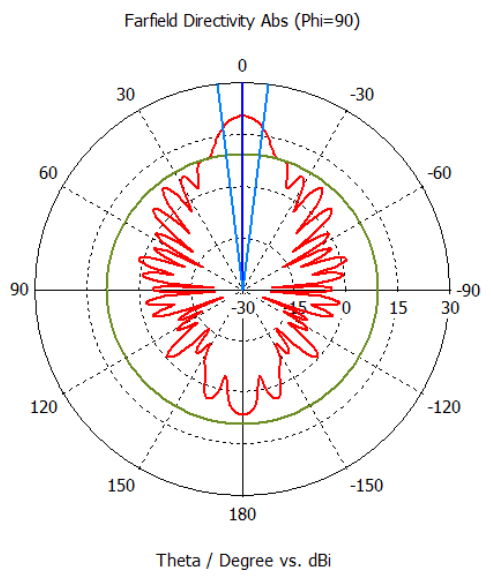

Fig. 12 Radiation pattern $2 \mathrm{D}$ plot at $\mathrm{f}=10 \mathrm{GHz}$ 


\section{Asia Pacific Journals}

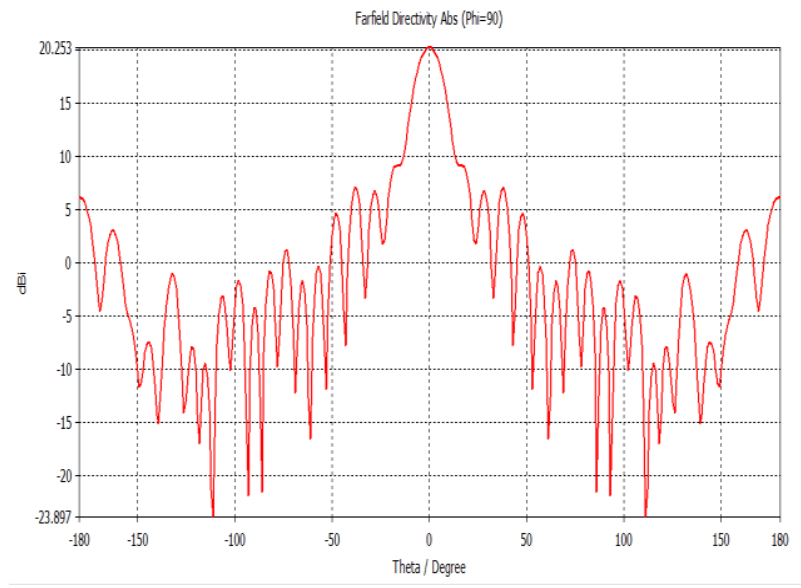

Fig. 13 Cartesian plot

TABLE III. COMPARISON OF HORN AND DIELECTRIC LOADED ANTENNA

\begin{tabular}{|c|c|c|c|}
\hline Antennas & $\begin{array}{c}\text { Directivity } \\
@ \\
\text { Air }=10 \mathrm{~cm} \\
\text { Soil }=10 \mathrm{~cm}\end{array}$ & $\begin{array}{c}\text { Directivity } \\
@ \\
\text { Air }=10 \mathrm{~cm} \\
\text { Soil }=30 \mathrm{~cm}\end{array}$ & $\begin{array}{c}\text { Directivity } \\
\text { Air }=10 \mathrm{~cm} \\
\text { Soil }=50 \mathrm{~cm}\end{array}$ \\
\hline Horn & $20.28 \mathrm{dBi}$ & $19.56 \mathrm{dBi}$ & $19.27 \mathrm{dBi}$ \\
\hline $\begin{array}{c}\text { Dielectric } \\
\text { loaded horn }\end{array}$ & $20.3 \mathrm{dBi}$ & $20.05 \mathrm{dBi}$ & $19.51 \mathrm{dBi}$ \\
\hline
\end{tabular}

Table III gives the comparison between horn and dielectric loaded horn antenna in terms of directivity. According to the simulation dielectric horn penetrates deeper into the soil for target detection. For varying depths of soil the directivity also varies and in each case dielectric horn has higher directivity in comparison to horn antenna. Hence dielectric horn has more penetration and can detect targets at larger depths in comparison to the horn and more suitable for GPR.

\section{CONCLUSION}

The paper presents horn and the dielectric loaded horn antennas in the frequency range $8 \mathrm{GHz}-12 \mathrm{GHz}$ using CST. Antennas with lower frequencies detect large objects at larger depths with lower resolution and higher penetration whereas high frequency antennas detect small objects at small depths with higher resolution and lower penetration. For GPR application antennas such as TEM horn, Vivaldi and Double ridge horn (DRH) are well suited. DRH gives the best solution with higher gain and better side lobe suppression.

\section{REFERENCES}

[1] Gitanjali Kalbhor, Manisha Vyas \& B. P. Patil "Antenna Design for Ground Penetrating Radar system". ISSN (Print) : 2278-8948, Volume2, Issue-6, 2013.

[2] A. G. Yarovoy and L. P. Ligthart, "Ultra-wideband antennas for ground penetrating radar," International Symposium on Antennas for Radar Earth Observation, 2000.

[3] Teggatz A., Jostingmeier A., Omar A, "The Imaging Capabilities of Ground Penetrating Radar for the Detection of Buried Anti-Personnel Landmines",2008.

[4] Disala Uduwawala, "A comprehensive study of resistor-loaded planar dipole antennas for ground penetrating radar",ISSN 1653-5146.

[5] Constantine A. Balanis "Antenna Theory, Analysis and Design", third edition ISBN: 0-471-66782-X. 